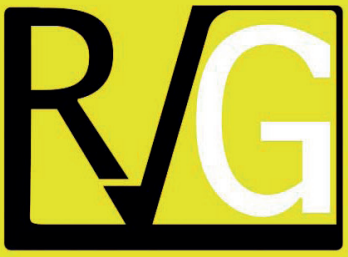

Julio - Septiembre, 2021

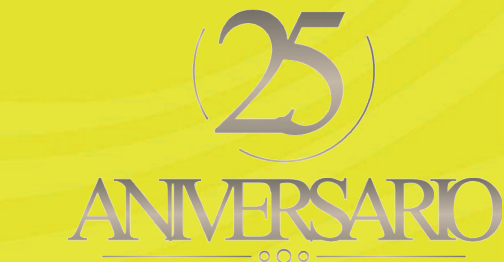

ANMERSARO
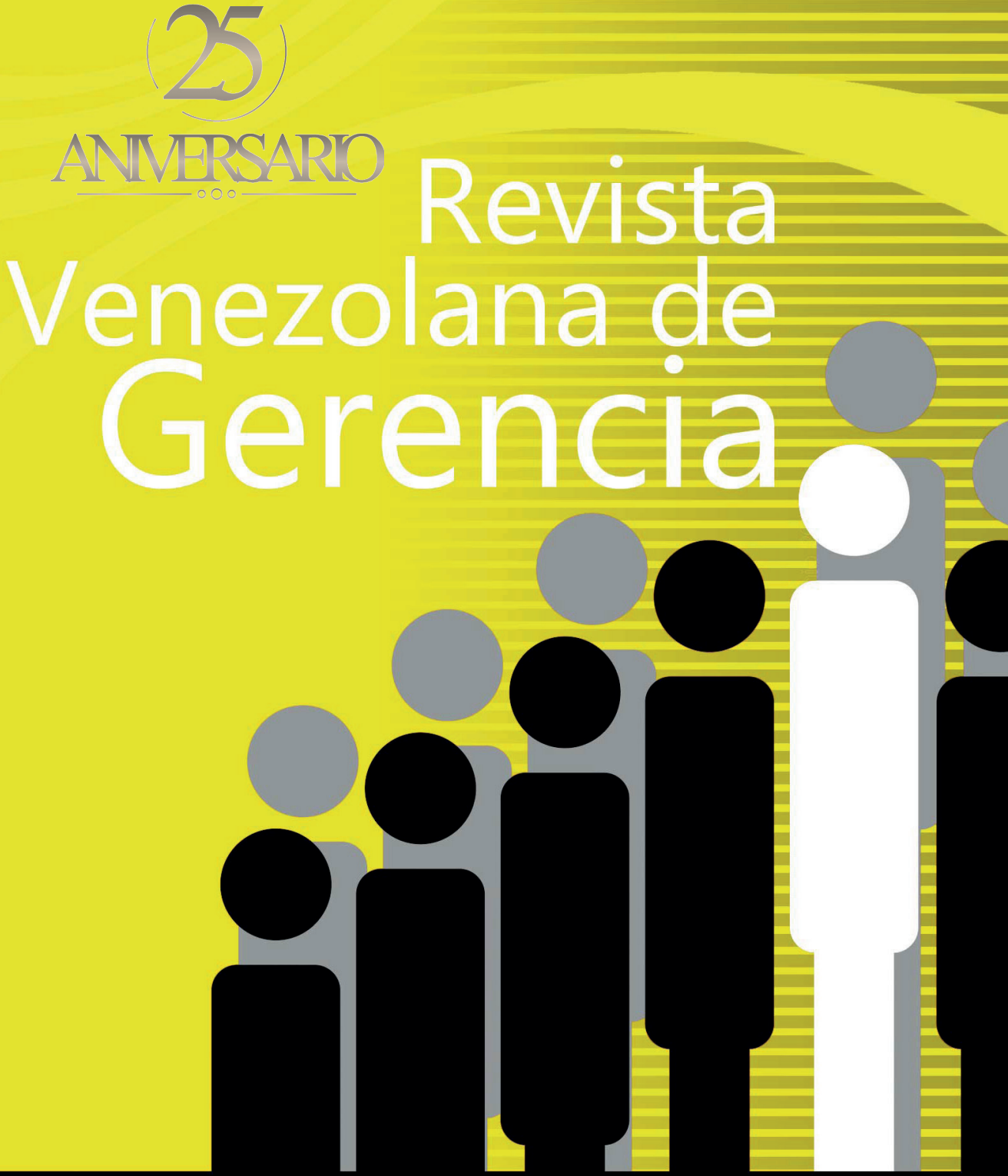

UNIVERSIDAD DEL ZULIA (LUZ)

Facultad de Ciencias Económicas y Sociales Centro de Estudios de la Empresa

ISSN 1315-99

Esta obra está bajo una licencia de Creative Comm Reconocimiento-NoComercial-Compartirlgual 3.0 Unpo http://creativecommons.org/licenses/by-nc-sa/3.0/deed.es 
COMO CITAR: Castañón Rodríguez, J. C., Baca Pumarejo, J. R., Macías Villarreal, J. C., y Villanueva Hernández, V. (2021). Red social Facebook como herramienta de marketing en micro, pequeñas y medianas empresas. Revista Venezolana de Gerencia (RVG), 26(95), 882-897. https://doi. org/10.52080/rvgluz.27.95.27
Universidad del Zulia (LUZ)

Revista Venezolana de Gerencia (RVG)

Año 26 No. 95 Julio-Septiembre 2021, 882-897

ISSN 1315-9984 / e-ISSN 2477-9423

\title{
Red social Facebook como herramienta de marketing en micro, pequeñas y medianas empresas
}

\author{
Castañón Rodríguez, Julio César* \\ Baca Pumarejo, José Rafael** \\ Macías Villarreal, Julio César*** \\ Villanueva Hernández, Vicente ${ }^{\star * * *}$
}

\section{Resumen}

El objetivo de la investigación fue analizar los esfuerzos de empresas para utilizar la red social Facebook. Se utilizaron datos de 60 micro, pequeñas y medianas empresas (en lo sucesivo mipymes) de los sectores económicos: Restaurantes, Boutiques, Refaccionarias e Imprentas en Ciudad (Cd.) Victoria Tamaulipas, México. Como indicadores de los esfuerzos en Facebook se determinaron tres variables: intensidad, riqueza y capacidad de respuesta. La metodología fue descriptiva y el tipo de muestreo fue por conveniencia, atendiendo al criterio de que las empresas tuvieran activo su perfil en Facebook desde hace 5 años o más La recolección de los datos se hizo

Aceptado: 10.05.21

* Maestro en Administración por la Universidad Autónoma de Tamaulipas, perfil PRODEP, estudiante del Doctorado en Ciencias Administrativas por la Universidad Autónoma de Tamaulipas, Profesor de Tiempo Completo de la Universidad Autónoma de Tamaulipas, adscrito a la Facultad de Comercio y Administración Victoria, e-mail: jcastanon@docentes.uat.edu.mx ORCID https://orcid.org/0000-0003-4396-9973

** Doctor en Educación Internacional por la Universidad Autónoma de Tamaulipas. Profesor Investigador de Tiempo Completo de la Universidad Autónoma de Tamaulipas, adscrito a la Facultad de Comercio y Administración Victoria. Perfil PRODEP. Pertenece al SNI Nivel 1. Línea de investigación: Nuevas tecnologías para la gestión del conocimiento, la competitividad y la innovación. Líder del Cuerpo Académico Consolidado "Nuevas Tecnología, Capital Humano y Competitividad", e-mail: rbaca@docentes.uat.edu.mx ORCID https:// orcid.org/0000-0002-7014-5446

*** Doctor en Ciencias de la Administración por la Universidad Internacional de América. Profesor de la Facultad de Comercio y Administración Victoria de la Universidad Autónoma de Tamaulipas. Línea de investigación: Nuevas tecnologías para la gestión del conocimiento, la competitividad y la innovación, e-mail: jemacias@. docentes.uat.edu.mx ORCID https://orcid.org/0000-0002-8636-0570

*** Doctor en Investigación Educativa por la Escuela Normal Superior de Ciudad Madero. Profesor de Tiempo Completo de la Unidad Académica Multidisciplinaria Reynosa Rodhe de la Universidad Autónoma de Tamaulipas. Línea de investigación: Nuevas tecnologías para la gestión del conocimiento, la competitividad y la innovación, e-mail: vhernand@docentes.uat.edu.mx ORCID https://orcid.org/0000-0002-9388-5746 
de las páginas de Facebook de las 60 empresas durante dos periodos, una primera fase (del 1 de marzo de 2018 al 31 de julio de 2018) y en una segunda fase (del 1 de septiembre del 2018 al 28 de febrero de 2019). Los resultados mostraron un nivel de intensidad de mercadotecnia digital poco desarrollado, una circunstancia que sugiere fortalecer y fomentar el uso de la red social mediante una capacitación adecuada y un enfoque operativo para elevar la intensidad, la riqueza y la capacidad de respuesta de las actividades de comunicación de mercadotecnia realizadas a través de facebook de estas empresas.

Palabras clave: Facebook; intensidad; riqueza; capacidad de respuesta; marketing digital.

\title{
The use of social network Facebook as a marketing tool in Cd. Victoria, Tamaulipas
}

\begin{abstract}
The objective of the research was to analyze the efforts of companies to use the social network Facebook, for which data from 60 micro, small and medium-sized companies (hereinafter MSMEs) of the economic sectors were used: Restaurants, Boutiques, Repair Shops and Printers in Ciudad (Cd.) Victoria Tamaulipas, Mexico. As indicators of the efforts in Facebook, three variables were determined: intensity, richness and responsiveness. The methodology was descriptive and the type of sampling was for convenience, taking into account the criteria that the companies had had their Facebook profile active for 5 years or more. The data was collected from the Facebook pages of the 60 companies for two periods, a first phase (from March 1, 2018 to July 31, 2018) and a second phase (from September 1, 2018 to February 28, 2019). The results show an underdeveloped level of intensity of digital marketing, a circumstance that suggests strengthening and promoting the use of the social network through adequate training and an operational approach to increase the intensity, richness and responsiveness of marketing activities. marketing communication made through facebook of these companies.
\end{abstract}

Keywords: Facebook; intensity; wealth; responsiveness; digital marketing.

\section{Introducción}

Las redes sociales, son una de las herramientas que representa la evolución de la web 4.0, impactan la forma en cómo las empresas innovan y protagonizan esta revolución que implica una interacción más intensa con los usuarios a través de la asistencia virtual y la inteligencia artificial; 
en consecuencia, la estrategia, la investigación, la práctica, la expansión de la comercialización y la comunicación organizacional experimenta un cambio esencial (Latorre, 2018).

En este sentido, las organizaciones en la actualidad sufren diversos cambios ante la globalización de la redes por lo que, según Aucay y Herrera (2017:82) señala que estas:

"han ocasionado cambios en los ámbitos interno y externo de las empresas, las mismas que deben adaptarse a entornos que cada día son más complejos y competitivos, donde los administradores deben poseer habilidad y experticia para crear estrategias de valor y para llegar a satisfacer a un nuevo estilo de demandantes, que influenciados por las publicaciones presentadas a través de los diferentes medios virtuales, están cada vez más informados; lo que repercute en una sociedad más exigente"

Esta tendencia es evidente porque en el último trimestre de 2020 , Facebook registró 2,449 millones de usuarios activos en todo el mundo ubicándose como la red social más popular, seguida de YouTube con 2,000 millones de usuarios, con el mayor número de seguidores jóvenes ( $76 \%$ de la población entre 16 y 30 años), y en tercer lugar, se registra Instagram con 1,000 millones de usuarios evidenciando una expansión creciente. Por su parte, Tik-Tok ostenta 800 millones de usuarios para colocarse en cuarto lugar; ubicándose Linkedln en quinto lugar, con 610 millones de usuarios y; en sexto y séptimo lugar, están Twitter con 339.6 y Pinterest con 322 millones de usuarios, respectivamente. Por los datos anteriores se evidencia la hegemonía de Facebook sobre las otras redes (Escuela de Negocios de la Innovación y los Emprendedores [IEBS], 2020).
En México, en el primer bimestre 2021 y en base a una población de 136.1 millones de habitantes, se registra $69 \%$ de la población con perfil en alguna de las redes sociales; en este contexto, la red con más uso es Facebook, con poco más de 78.70 millones de usuarios, le sigue Instagram con 24 millones de usuarios, Linkedln con 13 millones; mientras Twitter reune 9.5 millones de usuarios (Instituto Leonardo Bravo A.C. [iLB], 2021).

Esta hegemonía es explicable, inicialmente porque Facebook fue la primera red social del mundo y actualmente proporciona a las empresas la ventaja de diseñar su propio perfil y compartir información importante en la conformación de su comunidad de marca (a través de las ofertas, promociones y acciones de las firmas), permite también la capacidad de dirigirse a los usuarios y establecer una conversación bidireccional con los consumidores $y$, por tanto, generar una mayor confianza y fidelidad de los clientes (Algesheimer et al. 2005; Hennig-Thurau et al. 2010; Thompson y Sinha 2008). De esta manera los esfuerzos de las empresas en Facebook, pueden influir en las ventas de productos/servicios, en el conocimiento, las actitudes, las percepciones, las decisiones de compra y la fidelidad a la marca de los clientes potenciales y actuales al recibir los mensajes.

El valor empresarial de Facebook, ha sido investigado de manera reciente por estudios que analizan el grado en que las empresas se involucran con esta red social a través de las medidas y métricas que sirven para investigar su eficacia. Al examinar los resultados de investigación de las redes sociales llevadas a cabo por Goh et al. (2013), 
Miller y Tucker (2013), Rishika et al. (2013), Tirunillai y Tellis (2012), se deduce que el estudio queda incluido en aquellos que miden los esfuerzos al usar Facebook en las empresas (páginas de Facebook en nuestro caso).

En este sentido, el objetivo de esta investigación fue analizar los esfuerzos de empresas en Facebook mediante indicadores representados por tres dimensiones: intensidad, riqueza y capacidad de respuesta considerando la naturaleza de las publicaciones. También se determina si existen diferencias significativas entre los cuatro tipos de mipymes analizadas: boutiques, restaurantes, refaccionarias e imprentas en cuanto a sus esfuerzos en Facebook. Para lograr los objetivos de la investigación, se utilizaron datos de las páginas de Facebook de 60 empresas durante dos periodos, comprendidos en una primera fase, del 1 de marzo de 2018 al 31 de julio de 2018 y, en la segunda fase del 1 de septiembre del 2018 al 28 de febrero de 2019.

\section{Redes sociales: revisión de literatura}

Las redes sociales ocupan cada vez más el tiempo que los consumidores pasan conectados. La combinación de la comunicación de mercadotecnia con redes sociales es una parte importante del presupuesto de las empresas, a medida que aumentan su actividad en los medios sociales el papel que juegan los contenidos se ha vuelto más importante; por ello, la comunicación mercadotécnica busca desarrollar contenidos que sean atractivos para los públicos y fortalecer los objetivos de las organizaciones (Dokyun et al. 2018; Sánchez \& De la Garza, 2018).
Los medios sociales se pueden usar para lograr diversos objetivos organizacionales, entre estos, crear conciencia, aumentar las ventas o fidelizar, solo por mencionar algunos, estos pueden ser un lugar para las redes sociales, las reseñas de los consumidores, los blogs, las wikis y los foros de Internet con diversas plataformas. Estas últimas son un excelente ejemplo para conocer los perfiles de usuario, para la publicación y el comentario con el etiquetado de diversas formas de contenido (incluyendo texto, foto y video). Las plataformas de medios sociales se clasifican en varios foros en línea, como las redes sociales (Facebook y LinkedIn), los medios compartidos (YouTube, Flickr e Instagram), los blogs (Tumblr y Wordpress), los sitios web colaborativos (Wikipedia), los mundos virtuales (SecondLife), las comunidades comerciales (SnapDeal y PepperFry) y los marcadores sociales (StumbleUpon), entre otros (Mangold y Faulds, 2009). Estas plataformas influyen los comportamientos de los usuarios y sus decisiones en la vida real (Kietzmann et al. 2011).

Al respecto, la principal característica de los medios sociales es la interacción que se logra entre los actores, especialmente, vale la pena mencionar algunos estudios que destacan la versatilidad e interés que este tipo de interacción puede producir en las relaciones entre empresas y usuarios. De allí se desprenden estudios para medir la utilidad de los contenidos generados por los usuarios para las empresas llevados a cabo por Sawhney et al. (2005), Godes y Mayzlin (2009), investigaciones sobre las implicaciones teóricas de los esfuerzos de las empresas en las comunidades online 
realizado por Dellarocas (2006).

Así también, el estudio de Dokyun et al. (2018) describiendo el impacto del contenido publicitario en las redes sociales en relación al compromiso de los clientes utilizando datos de Facebook; en este caso, se codificaron 106,316 mensajes de 782 empresas, utilizando una combinación de Amazon MechanicalTurk y algoritmos de lenguaje natural, con estas herramientas se estudió la asociación de varios tipos de contenido de marketing en redes sociales con la participación de los usuarios.

En general, se observó que la inclusión de contenidos relacionados con rasgos de la personalidad de la marca, como el humor y la emoción, se asoció con mayores niveles de compromiso de los consumidores (Likes, comentarios y clics); adicional, se encontró que el contenido solo informativo, como los precios y las ofertas, refleja niveles menores de compromiso cuando los mensajes aparecen de forma aislada. Por otro lado, los resultados mostraron mayores beneficios cuando el contenido se combina con características informativas que ayudan a obtener clientes potenciales inmediatos (a través de clics mejorados); asimismo, el contenido relacionado con la personalidad de la marca ayuda a fortalecer la posición de esta en las redes sociales (a través de un compromiso mejorado) (Dokyun et al. 2018).

Hay que mencionar, que en las publicaciones de Facebook la valencia de la publicación y las características del contenido afectan el compromiso medido en función del número de $\mathrm{Me}$ gusta y de los comentarios recibidos por una publicación. Partiendo del análisis controlando de una variedad de factores, tales como, características lingüísticas de las publicaciones, las características de formato y la heterogeneidad del contexto, se evidencia que las publicaciones negativas generadas por el usuario en las páginas comerciales de Facebook, son significativamente más frecuentes que las positivas, lo que contrasta con la distribución de valencia de las reseñas de consumidores en línea (Yang et al. 2019)

Igualmente, el compromiso depende no solo de la valencia de una publicación, sino también de las formas específicas en las que la publicación es positiva o negativa, resaltando tres tipos de quejas de clientes, relacionados con la calidad de los productos y servicios, cuestiones económicas y sociales, y medioambientales. Como dato interesante, las quejas sociales reciben más me gusta pero menos comentarios que las quejas de calidad o precio; en este sentido, las publicaciones positivas tienden a atraer más me gusta pero menos comentarios que las publicaciones neutrales. En general, las publicaciones generadas por usuarios en las páginas comerciales de Facebook representan una forma distintiva de UGC (User Generated Content) que es conceptualmente diferente de las reseñas de consumidores en línea (Yang et al. 2019)

Complementariamente, también se han hecho análisis de los rendimientos financieros de las empresas a partir de las acciones de comunicación en una plataforma de redes sociales centrándose, específicamente, en páginas comerciales de Facebook, en este sentido, el volumen y la puntualidad de las respuestas de una empresa a los mensajes negativos de los clientes, están asociados con un aumento en la satisfacción del cliente y con un impacto en el desempeño de mercado de la empresa medido por rendimientos 
anormales; en contraste, las respuestas a los mensajes positivos no se asocian de manera significativa con el desempeño de la empresa (Chung et al. 2020).

Entonces, como resultado de los diversos estudios se han propuesto modelos empíricos para medir los esfuerzos de una organización en los medios sociales, sirviendo para orientar los abordajes investigativos de otros, resumiendo, se pueden puntualizar los que se orientan evaluar los esfuerzos (Vlachvei \& Notta, 2015; Öztamur \& Karakadılar, 2014); otros evalúan el impacto de la actividad de las empresas en las redes sociales en los resultados financieros y la reputación de la marca (Chung et al. 2020; Hong et al. 2016); y otros se esfuerzan en valorar el rendimiento de la inversión en marketing en las redes sociales (Hoffman \& Fodor, 2010).

Resalta, el compromiso de las empresas que se evalúa en función del número de "likes" o fans como señalan Paniagua y Sapena (2014); la intensidad y la calidad del contenido (fotos, videos, enlaces); así como, el número de las actualizaciones por periodo operativo, son claves para estimular la atención de los públicos objetivo (Chung et al. 2020; Galati et al. 2017; Hong et al. 2016; Karjaluoto et al. 2015; Moro et al. 2016; Öztamur \& Karakadılar, 2014; Vlachvei y Notta 2015).

Sin embargo, como señalan Öztamur \& Karakadılar (2014), las métricas como el número de páginas vistas, visitantes, amigos o seguidores no implican mayores pedidos 0 ventas. Estos autores, comparando las actividades de medios sociales de empresas estadounidenses y turcas en Facebook y Twitter, sugieren que las empresas deben dedicar tiempo a crear contenidos ricos en sus canales de medios sociales.

\section{Evaluación de los esfuerzos de las empresas en los medios sociales}

En general, las métricas más comunes relacionadas con Facebook son: 1) Número de likes; 2) Número de comentarios; 3) Número de compartidos (shares); 4) Naturaleza de la publicación y 5) Participación. (Sawhney et al. 2005; Godes \& Mayzlin 2009; Dellarocas 2006; Miller y Tucker, 2013; Chung et al. 2020). Particularmente, en este estudio se aborda las métricas de alcance y compromiso (Weber, 2011), a través de tres dimensiones de los esfuerzos de una empresa en una página de medios sociales: La intensidad de los esfuerzos de la empresa; b) La riqueza de los esfuerzos de la empresa y c) La capacidad de respuesta de los esfuerzos de la empresa.

Al respecto, la intensidad de los esfuerzos de la empresa se refiere al volumen de publicaciones y comentarios publicados por una empresa, con lo cual se espera lograr una mayor frecuencia de visualizaciones y acciones de los clientes y seguidores, lo cual incrementaría su compromiso e influiría en el valor de mercado de la empresa. La intensidad es una medida cuantitativa que caracteriza la actividad de Facebook y se obtiene al dividir la suma del número de publicaciones y comentarios en la página de Facebook de una empresa entre el número de seguidores de esa página.

Intensidad $=($ Posts + Comentarios $) /$ Total de Seguidores

Por su parte, la riqueza de los esfuerzos de la empresa, es decir, la riqueza informativa de los mensajes 
publicados por una empresa a través de diferentes recursos -textos, imágenes o vídeos- tienen diferentes capacidades para transmitir información (Daft, 2011). Los mensajes más ricos tienen más probabilidades de ser percibidos por los consumidores porque son más atractivos e informativos (Larkin \& Simon 1987; Park y Hopkins 1992; Emerson 2012). La riqueza como segunda dimensión representa cualitativamente las publicaciones hechas por las empresas $y$, por lo tanto, también la calidad de sus actividades en redes sociales, su resultado está determinado por el cociente del número de publicaciones que contienen videos, fotos o enlaces entre el número total de publicaciones.

Riqueza $=$ (fotos + videos + enlaces) / Total de publicaciones

Por último, la capacidad de respuesta de los esfuerzos de la empresa, es la medida en que una empresa responde a los mensajes de los consumidores. Se sustenta en que al aumentar la frecuencia de contenidos informativos e interacciones con los clientes las empresa logran aumentar su compromiso y el rendimiento (Luo y Zhang, 2013). Al respecto, la empresa genera contenidos informativos e interactúa de manera dinámica y se calcula por el cociente de los comentarios que la empresa realiza entre la suma de estos comentarios de la empresa con los comentarios de los seguidores, (Weber, 2011).

Capacidad de respuesta = [(Comentarios de la empresa)]/ [(Comentarios de la empresa) + (Comentarios de Seguidores)]

\section{Aproximaciones metodológicas del estudio}

Con el fin de analizar los esfuerzos en redes sociales de los perfiles de Facebook de las 60 mipymes de comercio y servicios de Cd. Victoria las cuales pertenecen a la clasificación de Restaurantes, Boutiques, Refaccionarias e Imprentas, se debe hacer mención de que no todas utilizan páginas de promoción en Facebook. La elección de las empresas fue por un muestreo de conveniencia y se siguió el criterio de que las seleccionadas tuvieran activo su perfil desde hace 5 años o más, además que sus parámetros coincidieran con la clasificación de mipyme. La razón para la elección de este tipo de empresas es que en Cd. Victoria no abunda la industria, sino que prepondera la actividad comercial y de servicios, representada por este grupo de empresas, y se caracterizan porque son de todos los sectores y no deben tener más de 10 empleados. El monto máximo de venta anuales es de 4 millones de pesos y el tope máximo es de 4.6 (Diario Oficial de la Federación, 2009) y en la actualidad en México dan empleo al 70 por ciento de la población económicamente activa (Instituto Nacional de Estadística, Geografía e Informática [INEGI], 2019).

En este proceso se recopiló información detallada sobre todas las actividades (publicaciones [Posts], comentarios y Me gusta [Like]) de las páginas oficiales de Facebook de las 60 empresas mipymes de los tipos boutiques, restaurantes, refaccionarias e imprentas, en una primera fase (del 1 de marzo de 2018 al 31 de julio de 2018) y en una segunda fase (del 1 de septiembre del 2018 al 28 de febrero de 2019), se generó la base de datos y se llevó a cabo el análisis y proceso de los datos durante 2019, ante las circunstancias especiales que ha representado la pandemia covid19 la publicación se demoró el año 2020 pero 
a la vez hizo definitiva y única la base de datos, dado que la actualidad constituye un contexto totalmente diferente al que imperó en la labor de campo 2018-2019.

Los datos de Facebook se recolectaron a través del programa Next Analytics (Nextanalytics.com). Por lo que se recabaron los datos disponibles mediante Facebook para cada empresa. Se eligieron las métricas más utilizadas por las investigaciones revisadas las que se describen a detalle a continuación: m1) Número de likes. El ratio de likes se obtiene como un cociente de los likes del post entre el alcance total. m2) Número de comentarios. Es la suma total de comentarios que recibe un post de la página. El ratio de comentarios se calcula como el cociente de los comentarios del post entre el alcance total del mismo. m3) Número de compartidos (shares). El número de compartidos es el total de compartidos que ha recibido un post. El ratio de compartidos es el cociente del número total de compartidos de la publicación entre el alcance total de la misma. m4) Naturaleza de la publicación. Las publicaciones más comunes son: una actualización de estado, una foto, un enlace (a una URL), un estado de vídeo (video descargado de alguna plataforma).

Asimismo, se incluyó, m5) Participación, según la propia red social Facebook se trata el número de personas que ha hecho clic en cualquier parte de una publicación, señalando que le gusta, comentando o compartiendo la publicación en cuestión. El ratio de compromiso se calcula como el cociente del compromiso entre el alcance total. Todos estos ratios mencionados se expresan en decimales o bien en porcentajes.

Las métricas más importantes de los medios sociales de acuerdo con Weber (2011) son el alcance, el compromiso y el negocio. En el caso de las comunidades de Facebook, el interés se centra en las métricas de alcance y compromiso. Por lo que para evaluar los esfuerzos de las empresas que se analizan, se han identificado tres dimensiones de los esfuerzos de una empresa en medios sociales, (Galati et al. 2017 y Vlachvei \& Notta, 2015):

Estas dimensiones calculadas mediante cocientes mencionados se expresan en decimales 0 bien en porcentaje $y$, en base a estas dimensiones, se formularon preguntas de Investigación orientadoras del camino a seguir para cumplir el objetivo de analizar los esfuerzos de las empresas en sus páginas de Facebook de las empresas de los 4 grupos de mipymes de comercio y servicios de $\mathrm{Cd}$. Victoria Tamaulipas, México. Las preguntas de investigación, en este sentido quedaron estructuradas de la siguiente forma: 1) ¿Cuál es el nivel de la Intensidad que manifiestan las páginas de Facebook de las mipymes de Cd. Victoria, Tamaulipas?, 2) ¿Cuál es el nivel de la Riqueza que manifiestan las páginas de Facebook de las mipymes de Cd. Victoria, Tamaulipas?, 3) ¿Cuál es el nivel de la Capacidad de respuesta de las páginas de Facebook de las mipymes de Cd. Victoria, Tamaulipas?

\section{Esfuerzos de mipymes de comercio y servicios de $\mathrm{Cd}$ Victoria en sus páginas de Facebook: Resultados}

Una vez finalizado el proceso de recolección, captura y análisis de datos referentes a las 60 mipymes ubicadas en Ciudad Victoria, Tamaulipas, se resumen a continuación los resultados más importantes en relación con el objetivo 
Castañón Rodríguez, Julio César; Baca Pumarejo, José Rafael; Macías Villarreal, Julio César; Villanueva Hernández, Vicente

Red social Facebook como herramienta de marketing en Cd. Victoria, Tamaulipas

de investigación.

La Tabla 1 presenta los datos descriptivos sobre la actividad en Facebook de las mipymes de la muestra. El promedio de posts/día es relativamente pequeño y alcanza sólo 0.50 posts diarios para el periodo de la muestra; sin embargo, algunas empresas publican como máximo 2 posts al día. La media de likes por post es de 38.79 , mientras que la media de comentarios por post es de 16.28 y en promedio se registran 0.85 acciones de compartición (shares) por cada post. El promedio de fans de las 60 empresas es de 25,004 fans, mientras que hay una empresa con más de 1,105,377 fans.

\section{Tabla 1}

\section{Datos descriptivos de los esfuerzos en Facebook de 60 mipymes de Comercio y Servicios de Cd. Victoria, Tam.}

\begin{tabular}{lcccc}
\hline Indicador & Promedio & Min & Max & Desviación estándar \\
\hline Posts/día & 0.508333 & 0.1 & 2.26667 & 0.382755644 \\
\hline Likes/post & 38.79117 & 0.333 & 441.2 & 81.40104401 \\
\hline Comentarios/post & 16.28527 & 0 & 184.833 & 41.27237839 \\
\hline Shares/post & 0.8514 & 0 & 6.577 & 1.246754093 \\
Seguidores & 25004.03 & 136 & 1105377 & 142165.7862 \\
\hline
\end{tabular}

Fuente: Elaboración propia a partir de la información recolectada

Se dividió la muestra en los grupos según el tipo de las mipymes, con el objetivo de establecer los niveles de los esfuerzos en Facebook según sus características. De forma descriptiva, la Tabla 2 presenta los datos de los cuatro tipos de mipymes: Boutiques, Restaurantes, Refaccionarias e Imprentas.

Según la evidencia los promedios de posts por día y likes por post, denota que las Boutiques y Restaurantes llevan a cabo los mayores esfuerzos de Facebook porque el promedio de posts/ día de 0.67 y 0.55 respectivamente aunados a sus promedios de likes/ post de 33.17 y 70.79 que lo confirman, mientras que las refaccionarias y las imprentas presentan valores promedios inferiores a la media de la muestra de estas mipymes. En contraste, respecto a comentarios por post, los resultados son diferentes ya que las empresas Restaurantes y Refaccionarias alcanzan en promedio 37.87 y 16.65 comentarios por post respectivamente, superiores a la media de la muestra de las mipymes consideradas (14.32). En cuanto a las comparticiones o shares por post, las empresas del tipo boutiques e imprentas, 
en promedio tienen 1.26 y 1.08 shares por post respectivamente los cuales son mayores a la media de la muestra de las mipymes (.79).

\section{Tabla 2}

Promedio de Likes, Comentarios y Shares por post, y riqueza para cada grupo de mipymes de Comercio y servicios de Cd. Victoria.

\begin{tabular}{|c|c|c|c|c|c|c|}
\hline Mipymes & $\begin{array}{l}\text { Núm. de } \\
\text { empresas }\end{array}$ & Post/día & Likes/post & $\begin{array}{l}\text { Comentarios/ } \\
\text { post }\end{array}$ & Shares/post & Riqueza \\
\hline Boutiques & 20 & 0.6733 & 33.17 & 2.5 & 1.26 & 0.900 \\
\hline Restaurantes & 20 & 0.5533 & 70.79 & 37.87 & 0.6482 & 0.085 \\
\hline Refaccionarias & 10 & 0.32 & 20.96 & 16.65 & 0.1957 & 0.170 \\
\hline Imprentas & 10 & 0.2766 & 3.851 & 0.2793 & 1.0864 & 0.254 \\
\hline
\end{tabular}

Fuente: Elaboración propia a partir de la información recolectada

La Tabla 3 describe como las mipymes de comercio y servicios de la muestra tienen preferencia a presentar a sus seguidores de Facebook fotos $(3.88 \%$ de los posts), es decir, las empresas transfieren información a sus usuarios principalmente mediante el uso de fotos, ya que en promedio se encontraron 3.88 fotos por cada publicación, lo que sugiere que se suben aproximadamente
4 fotos (que van desde un mínimo 0.15 a un máximo de 54.25), la desviación estándar fue de 8.48 valor que se califica como modesto, es decir las 60 empresas de la muestra variaron muy poco el promedio de 4 fotos por publicación algunas más, algunas menos, evidencia que no contribuye a un nivel de riqueza de contenidos competitiva.

\section{Tabla 3}

Naturaleza de los posts para las mipymes de Comercio y servicios de Cd. Victoria

\begin{tabular}{lllll}
\hline Indicador & Promedio & Min & Max & Desviación Estándar \\
\hline Fotos/post & 3.8883 & 0.6 & 54.25 & 8.480653907 \\
Videos/post & 0.069733333 & 0 & 0.578 & 0.108149436 \\
Enlaces/post & 3.2082 & 0 & 177 & 22.82072035 \\
\hline
\end{tabular}

Fuente: Elaboración propia a partir de la información recolectada

En el caso de los videos publicados por las empresas, el promedio estuvo muy por debajo de los otros dos indicadores, ya que tuvo un promedio de
0.06 con valores promedio de mínimo de 0 y máximo de 0.57 y esta tendencia es confirmada por el valor poco significativo de su desviación estándar igual a 0.10 . 
Castañón Rodríguez, Julio César; Baca Pumarejo, José Rafael; Macías Villarreal, Julio César; Villanueva Hernández, Vicente

Red social Facebook como herramienta de marketing en Cd. Victoria, Tamaulipas

Los enlaces a información adicional tuvieron un promedio muy cercano al de las fotos el cual fue de 3.20 , donde el número de enlaces en promedio varió de un mínimo de 0 hasta un valor máximo de 177. En este indicador el valor de la desviación estándar fue de 22.82 valor mayor comparado con las de los otros dos indicadores, lo que sugiere que habrá empresas que rebasen el promedio de 3 enlaces por publicación.
Asimismo, la Tabla 4 muestra que las imprentas prefieren informar a través de publicaciones con fotos (una media del $86.64 \%$ del total de publicaciones) y muy raramente a través de textos $(4.61 \%)$ y de vídeos $(2.39 \%)$, para el caso de las Boutiques el uso de enlaces es importante $(50.26 \%)$ al igual que las fotos $(50.26 \%)$, especialmente si se compara con la media de enlaces que es del $26.33 \%$.

\section{Tabla 4}

Tipo de posts por cada grupo de mipymes de Comercio y servicios de $\mathrm{Cd}$. Victoria

\begin{tabular}{lllll}
\hline & Boutiques & Restaurantes & Refaccionarias & Imprentas \\
\hline Fotos/post & 0.5026 & 0.6876 & 0.7586 & 0.8664 \\
\hline Enlaces/post & 0.5026 & 0.2335 & 0.2077 & 0.1095 \\
\hline Texto/posts & 0,0650 & 0,04543 & 0,0361 & 0,0461 \\
\hline $\begin{array}{l}\text { Vídeos/post } \\
0.0024\end{array}$ & & & & \\
0.07885 & & & & \\
0.0335 & & & & \\
\hline
\end{tabular}

Fuente: Elaboración propia a partir de la información recolectada

Como se muestra en la Tabla 5 , por término medio, una empresa comenta o publica1.82 veces al día; aproximadamente el $35.22 \%$ de estas publicaciones contiene información enriquecida; con mejor valoración se destaca que las empresas interactúan con los usuarios en $63.17 \%$, a través de los comentarios de respuesta. 


\begin{tabular}{|c|c|c|c|c|}
\hline & media & Desviación estándar & $\max$ & $\min$ \\
\hline Intensidad & 1.82870 & .1879 & 0.6735 & 0.026 \\
\hline Riqueza & 0.35225 & 0.3716 & 0.9 & 0.085 \\
\hline $\begin{array}{l}\text { Capacidad de res- } \\
\text { puesta }\end{array}$ & 0.63175 & 0.3447 & 0.9674 & 0.304 \\
\hline
\end{tabular}

Fuente: Elaboración propia a partir de la información recolectada

En síntesis, los resultados evidenciaron que las empresas han adoptado las nuevas tendencias para la promoción de mercadotecnia; sin embargo, se aprecia una actividad en la red social Facebook limitada dado que las publicaciones exhibieron un nivel modesto, tanto de riqueza como de contenido, particularmente, en cuanto a la subida de fotos y videos, así como, lo relacionado a una buena interacción con los usuarios. Si bien se percibió que se han involucrado en redes sociales, a través de los valores de intensidad y riqueza de manera incipiente, su alcance es conservador.

Los resultados muestran que, por término medio, una empresa comenta o publica 1.82 veces al día, mientras que se debe enfatizar que solo el $35.22 \%$ de ellas contienen información enriquecida, lo que significa que este sector de empresas debe redoblar esfuerzos para ofrecer información enriquecida a través de Facebook y responder con más compromiso y de forma activa a los mensajes de los consumidores. A partir de los resultados es evidente que el bajo costo de esta herramienta de comunicación, en comparación con los medios tradicionales, pareciera restar importancia a su uso; no obstante, las empresas están desaprovechando el recurso como un canal estratégico en estos tiempos de virtualidad.

\section{Conclusiones}

La web ha demostrado ser, aparte de un instrumento de información valiosa, un vehículo bien equipado de técnicas de mercadotecnia modernas y rentables, en especial, para la mipymes de comercio y servicios.

Resulta importante desarrollar, mantener e innovar un perfil de Facebook actualizado y activo, en el análisis de los esfuerzos de las empresas para usar la red social Facebook, que aún no se ha logrado impulsar una verdadera mercadotecnia digital, concibiendo su uso solo como una moda o por deber, es decir, por la necesidad de estar presentes en el mundo digital; pero, no porque exista una verdadera estrategia de comunicación de marketing que potencie las relaciones con los clientes.

El análisis de los esfuerzos de Facebook de las empresas en cuatro sectores de la economía mexicana, 
Castañón Rodríguez, Julio César; Baca Pumarejo, José Rafael; Macías Villarreal, Julio César; Villanueva Hernández, Vicente

Red social Facebook como herramienta de marketing en Cd. Victoria, Tamaulipas

permite identificar los niveles de uso y aplicación de las redes sociales, la evidencia muestra también que las mipymes han incursionado en este ámbito avanzando el primer escalón; sin embargo, es esencial que se mantenga este ritmo mediante el seguimiento e incorporación de algunos estándares.

Las evidencias generadas en la investigación, y acorde a los resultados de otras investigaciones, se sugiere contratar personas especializadas y capacitadas en la operación de la red social Facebook, lo que sin duda favorece su expansión en el mercado local, regional o global. Porque la operación y mantenimiento de la red social Facebook contribuye al cuidado de la calidad del contenido de los mensajes, de las publicaciones, el estilo de escritura y la oportunidad del intercambio de información, factores clave que influyen en la lealtad del cliente y las ventas.

Las mipymes tienen una gran oportunidad con el uso de los medios sociales; sin embargo, hasta ahora, no han sabido explotar apropiadamente los beneficios de su uso, razón por la cual, la red social Facebook, como una plataforma para las acciones de mercadotecnia de la mipymes de Ciudad Victoria, Tamaulipas, México, debe concebirse de manera estratégica no se trata solo de tener presencia en la red, consiste en alcanzar valores de intensidad, riqueza y capacidad de respuesta que logren generar interacciones que se conviertan en el corto, mediano y largo plazo en fidelidad, confianza y sobre todo en información valiosa para orientar las estrategias que las empresas deben llevar a cabo en los medios sociales.

\section{Referencias bibliográficas}

Algesheimer, R., Dholakia, U., \& Herrmann, A. (2005). The Social Influence of Brand Community: Evidence from European Car Clubs. Journal of Marketing, 69(3), 1934. https://journals.sagepub.com/ doi/10.1509/jmkg.69.3.19.66363

Aucay Piedra, E., y Herrera Torres, P. (2017). Nivel de uso de las redes sociales en el proceso de comunicación en las MIPES de Cuenca. Retos, 7(14). https://doi. org/10.17163/ret.n14.2017.04

Chung, S., Animesh, A., Han, K., and Pinsonneault, A. (2020). Financial Returns to Firms' Communication Actionson Firm-Initiated Social Media: Evidence from Facebook Business Pages. Information Systems Research, 1-28. https://doi. org/10.1287/isre.2019.0884

Daft, R. (2011). Organization Theory and Design. 10ma. Ed. Cengage Learning Editores.

Dellarocas, C. (2006). Strategic manipulation of Internet opinion forums: Implications for consumers and firms. Management Science, 52(10), 1577-1593. https://doi. org/10.1287/mnsc. 1060.0567

Diario Oficial de la Federación (25 de junio de 2009). ABcuerdo por el que se establece la estratificación de las micro, pequeñas y medianas empresas. México, D.F., México. https://www.economia.gob.mx/files/ marco normativo/A539.pdf

Dokyun, L., Kartik, H., \& Harikesh, S. N. (2018). Advertising Content and Consumer Engagementon Social. Management Science, 1-27. https:// doi.org/10.1287/mnsc.2017.2902

Emerson, M. F. (2012). Social media marketing from A to $Z$. The New York Times. https://boss.blogs.nytimes. com/2012/12/07/social-media- 


\section{marketing-from-a-to-z/}

Escuela de Negocios de la Innovación y los Emprendedores IEBS (2020). Las Redes Sociales más utilizadas: cifras y estadísticas. https://www. iebschool.com/blog/medios-socialesmas-utilizadas-redes-sociales/

Galati, A., Crescimanno, M., Tinervia, S., \& Fagnani, F., (2017). Social media as a strategic marketing tool in the Sicilian wine industry: Evidence from Facebook. Wine Economics and Policy, 6(1), 40-47. https://www. sciencedirect.com/science/article/pii/ $\underline{\mathrm{S} 2212977417300054}$

Godes, D., \& Mayzlin, D. (2009). Firm-Created Word-of-Mouth Communication: Evidence from a Field Test. Marketing Science, 28(4), 721-739. https://msbfile03.usc.edu/ digitalmeasures/mayzlin/intellcont/ godes mayzlin 09-1.pdf

Goh, K. Y., Heng, C.-S., and Lin, Z. (2012). Social Media Brand Community and Consumer Behavior: Quantifying the Relative Impact of User- and Marketer-Generated Content. Information Systems Research, 24(1). http://dx.doi. org/10.2139/ssrn.2048614

Hennig-Thurau, T., Malthouse, E., Friege, Ch., Gensler, S., Lobschat, L., Rangaswamy, A., \& Skiera, B. (2010). The Impact of New Media on Customer Relationships. Journal of Service Research, 13(3), 311-330. https://doi. org/10.1177/1094670510375460

Hoffman, D., \& Fodor, M. (2010). Can You Measure the ROI of Your Social Media Marketing? MIT Sloan Manag., 52(1), 41-49. https://sloanreview.mit. edu/article/can-you-measure-the-roiof-your-social-media-marketing/

Hong, Y. J., Shin, D., \& Kim, J. H. (2016). High/low reputation companies dialogic communication activities and semantic networks on Facebook: A comparative study. Technological Forecasting and Social Change, Elsevier, 110(C), 78-92. https:// ideas.repec.org/a/eee/tefoso/ v110y2016icp78-92.html

Instituto Leonardo Bravo A.C. iLB. (2021). ¿Cuáles son las redes sociales más utilizadas en México en 2021? https://ilifebelt.com/redessociales-mas-utilizadas-en-mexicoen-2021/2020/11/

Instituto Nacional de Estadística, Geografía e Informática INEGI. (2019). Resultados de la Encuesta Nacional sobre Productividad y Competitividad de las Micro, Pequeñas y Medianas Empresas (ENAPROCE) 2018. https:// www.inegi.org.mx/contenidos/ saladeprensa/boletines/2019/ especiales/ENAPROCE2018.pdf

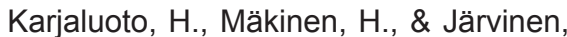
J. (2015). A Firm's Activity in Social Media and Its Relationship with Corporate Reputation and Firm Performance. 28 th Bled eConference. https://bit.ly/2TiNHVq

Kietzmann, J., Hermkens, K., McCarthy, I. P., \& Silvestre, B. (2011). Social media? Get serious! Understanding the functional building blocks of social media. Business Horizons, 54(3), 241-251. https://doi.org/10.1016/j. bushor.2011.01.005

Larkin, J. H. \& Simon, H. (1987). Why a diagramis (sometimes) worth ten thousand words. Cognitive Science, 11(1), 65-100. https://doi. org/10.1016/S0364-0213(87)80026$\underline{5}$

Latorre, M. (2018). Historia de las Web 1.0, 2.0, 3.0 y 4.0. Santiago de Surco: Universidad Marcelino Champagnat. https://umch.edu.pe/ arch/hnomarino/74 Historia $\% 20$ de\%20la\%20Web.pdf 
Castañón Rodríguez, Julio César; Baca Pumarejo, José Rafael; Macías Villarreal, Julio César; Villanueva Hernández, Vicente

Red social Facebook como herramienta de marketing en Cd. Victoria, Tamaulipas

Luo, X., \& Zhang, J. (2013). How Do Consumer Buzz and Traffic in Social Media Marketing Predict the Value of the Firm? Journal of Management Information Systems, 30(2), 213-238. https://doi.org/10.2753/MIS0742$\underline{1222300208}$

Mangold, W., \& Faulds, D. (2009). Social media: The new hybrid element of the promotion mix. Business Horizons, 52(4), 351-365. https://doi. org/10.1016/j.bushor.2009.03.002

Miller, Amalia R.\& Tucker, Catherine (2013). Active Social Media Management: The Case of Health Care. Information Systems Research, 24(1), 52-70. https://www.jstor.org/ stable/42004269

Moro, S., Rita, P., \& Vala, B., (2016). Predicting social media performance metrics and evaluation of the impact on brand building: A data mining approach. Journal of Business Research, 69(9), 3341$3351 . \quad$ https://doi.org/10.1016/j. jbusres.2016.02.010

Öztamur, D., \& Karakadılar, I. S. (2014). Exploring the Role of Social Media for SMEs: As a New Marketing Strategy Tool for the Firm Performance. Procedia - Social and Behavioral Sciences, 150(15) 511-520. https://doi.org/10.1016/i. sbspro.2014.09.067

Paniagua, J., \& Sapena, J. S. (2014). Business performance and social media: Love or hate? Bus. Horiz., 57(6), 719-728. https://doi. org/10.1016/j.bushor.2014.07.005

Park, Ok-Ch. \& Hopkins, R. (1992). Instructional conditions for using dynamic visual displays: a review. Instructional Science, 427$449 . \quad$ https://link.springer.com/ article/10.1007/BF00118557

Rishika, R., Kumar, A., Janakiraman,
R., \& Bezawada, R. (2013). The Effect of Customers' Social Media Participation on Customer Visit Frequency and Profitability: An Empirical Investigation. Information Systems Research, 24(1), 108$127 . \quad$ https://doi.org/10.1287/ isre.1120.0460

Sánchez Limón, M. L., \& De la Garza Cárdenas, M. H. (2018). Tecnologías de información y desempeño organizacional de las pymes del noreste de México. Revista Venezolana De Gerencia, 23(82), 298-313. https://doi.org/10.37960/ revista.v23i82.23750

Sawhney, M., Verona, G., \& Prandelli, E. (2005). Collaborating to create: The Internet as a platform for customer engagement in product innovation. Journal of interactive marketing, 19(4), 4-47. https://doi.org/10.1002/ dir. 20046

Thompson, S., \& Sinha, R. (2008). Brand Communities and New Product Adoption: The Influence and Limits of Oppositional Loyalty. Journal of Marketing, 72(6), 65-80. https://doi. org/10.1509/jmkg.72.6.065

Tirunillai, S., \& Tellis, G. (2012). Does Chatter Really Matter? Dynamics of User-Generated Content and Stock Performance. Marketing Science, 31(2), 198-215. https://www.jstor.org/ stable/41488019

Vlachvei, A., \& Notta, O. (2015). Greek Food Manufacturing Firms' Social Media Efforts: Evidence from Facebook. Procedia - Social and Behavioral Sciences, 308313. https://doi.org/10.1016/j. sbspro.2015.01.1205

Weber, L. (2011). A Comprehensive Digital Business Strategy for the Social Media Era. John Wiley \& Sons inc. 
pp. $882-897$

Revista Venezolana de Gerencia, Año 26 No. 95, 2021

Yang, M., Ren, Y., \& Adomavicius, G. (2019). Understanding UserGenerated Content and Customer
Engagement on Facebbok Business Pages. Information Systems Research, 1-17. https://doi. org/10.1287/isre.2019.0834 